\title{
The oxytocin/vasopressin receptor family has at least five members in the gnathostome lineage, including two distinct V2 subtypes
}

General and Comparative Endocrinology 175(1): 135-143

doi:10.1016/j.ygcen.2011.10.011

Accepted October 20, 2011

E-pub October 28, 2012

Published January 1, 2012

Figshare

doi:10.6084/m9.figshare.811860.

Shared October 1, 2013

Daniel Ocampo Daza*, Michalina Lewicka ${ }^{1}$, Dan Larhammar

Department of Neuroscience, Science for Life Laboratory, Uppsala Universitet, Box 593, SE-751 24

Uppsala, Sweden

* Corresponding author. E-mail address: Daniel.Ocampo-Daza@neuro.uu.se

${ }^{1}$ Current address: Department of Neuroscience, Karolinska Institutet, SE-171 77 Stockholm, Sweden

Cite as D. Ocampo Daza, M. Lewicka and D. Larhammar. The oxytocin/vasopressin family has at least five members in the gnathostome lineage, including two distinct V2 subtypes. General and Comparative Endocrinology, 175 (1) (2012) 135-143.

This document corresponds to the article as it appeared upon acceptance. You are free to download, print and distribute it for any purposes under a Creative Commons Attribution 3.0 Unported License (http://creativecommons.org/licenses/by/3.0/), provided the original work is cited as specified.

Errata: The introduction incorrectly states that "the V2 receptor inhibits adenylyl cyclase, thereby reducing the production of CAMP" on page 3. In fact the V2-type vasopressin receptors stimulate adenylyl cyclase and increase the cytosolic cyclic AMP release, see for instance Schöneberg et al., Molecular aspects of vasopressin receptor function, Advances in experimental medicine and biology 449 (1998) 347-58. This mistake was reported in the proofreading phase of pre-publication, but the correction was not carried to the final version of the article. The first paragraph of the discussion on page 9 suggests that all five identified OT/VP receptor subtypes are present in the anole lizard, frog and teleost fishes. In fact, teleost fishes lack one of the receptor subtypes, V1B. This does not affect the conclusions. 


\begin{abstract}
The vertebrate oxytocin and vasopressin receptors form a family of G-protein-coupled receptors (GPCRs) that mediate a large variety of functions, including social behavior and the regulation of blood pressure, water balance and reproduction. In mammals four family members have been identified, three of which respond to vasopressin (VP) named V1A, V1B and V2, and one of which is activated by oxytocin (OT), called the OT receptor. Four receptors have been identified in chicken as well, but these have received different names. Until recently only V1-type receptors have been described in several species of teleost fishes. We have identified family members in several gnathostome genomes and performed phylogenetic analyses to classify OT/VP- receptors across species and determine orthology relationships. Our phylogenetic tree identifies five distinct ancestral gnathostome receptor subtypes in the OT/VP receptor family: V1A, V1B, V2A, $\mathrm{V} 2 \mathrm{~B}$ and $\mathrm{OT}$ receptors. The existence of distinct $\mathrm{V} 2 \mathrm{~A}$ and $\mathrm{V} 2 \mathrm{~B}$ receptors has not been previously recognized. We have found these two subtypes in all examined teleost genomes as well as in available frog and lizard genomes and conclude that the V2A-type is orthologous to mammalian V2 receptors whereas the V2B-type is orthologous to avian V2 receptors. Some teleost fishes have acquired additional and more recent gene duplicates with up to eight receptor family members. Thus, this analysis reveals an unprecedented complexity in the gnathostome repertoire of OT/VP receptors, opening interesting research avenues regarding functions such as regulation of water balance, reproduction and behavior, particularly in reptiles, amphibians, teleost fishes and cartilaginous fishes.
\end{abstract}

Keywords: vasopressin receptor, vasotocin receptor, oxytocin receptor, mesotocin receptor, isotocin receptor; evolution; GPCR

Abbreviations: ICL3, third intracellular loop; NJ, neighbor joining; NNI, Nearest neighbor interchange; PhyML, phylogenetic maximum likelihood; SPR, subtree pruning and regrafting; TMHMM, transmembrane helix hidden Markov model. 


\section{Introduction}

The classical neuroendocrine nonapeptides vasopressin and oxytocin, released from hypothalamic neurons that extend their axons into the posterior pituitary, have profound effects on salt and water balance and reproduction, respectively $[\underline{6}, \underline{9}, \underline{32}]$. In addition, their widespread distribution in the brain in mammals correlates with important roles as neuropeptides influencing social behavior $[11,20]$ including social olfactory memory in mice [12] and trust and generosity in humans $[28,53]$. In fish the two peptides have been found to influence aggressive behavior $[\underline{4}, \underline{40}, \underline{49}]$. During the past few years, genetic variants of the peptide receptors have been found to correlate with variation in different aspects of human social interaction, see for instance [51].

Oxytocin and vasopressin were two of the first peptides that were sequenced in different classes of vertebrates. Unfortunately, each new sequence variant was given a separate name such as isotocin and mesotocin [2] for peptides that we now know are orthologs of mammalian oxytocin. It has also become clear that vasotocin in non-mammalian vertebrates is the ortholog of mammalian vasopressin. In fact, oxytocin and vasopressin arose from a common ancestral gene by a local duplication in a gnathostome ancestor, and the separate oxytocin and vasopressin lineages arose in early vertebrate evolution $[16,17]$. This makes other names for these peptides in the gnathostomes redundant in an evolutionary perspective, and also establishes a likely evolutionary time frame for the divergence of oxytocin and vasopressin systems, including the corresponding receptors.

The peptides exert their actions by activating a family of G-protein-coupled receptors (GPCR) belonging to the rhodopsin clan. In mammals, four receptors were cloned in the 1990s: one oxytocin receptor called OT with the gene name OXTR [24], and three vasopressin receptors named $\mathrm{V}_{1 \mathrm{~A}}(A V P R 1 A), \mathrm{V}_{\mathrm{B}}(A V P R 1 B)$ and $\mathrm{V}_{2}(A V P R 2)[7,44,46]$. The OT receptor (we will use the abbreviation OTR to distinguish the receptor from the OT peptide) and the two $V_{1}$ receptors primarily stimulate $\mathrm{Gq}$ and the $\mathrm{DAG} / \mathrm{IP} 3 / \mathrm{Ca}^{2+}$ pathway whereas the $\mathrm{V}_{2}$ receptor inhibits adenylyl cyclase, thereby reducing the production of CAMP. The four receptors have approx. $36-46 \%$ overall identity to each other in human, and $42-57 \%$ in the region spanning transmembrane helix (TM) 1 to TM7.

Over the past years, OT/VP receptors have been cloned and characterized from several species other than mammals, such as chicken, amphibians and ray-finned fishes. However, their orthology or paralogy relationships with the mammalian receptors and each other have not always been clear, although this issue has been addressed before. For example: the chicken vasotocin (vasopressin) receptor initially named VT1 [45] is clearly more similar to mammalian V2, although phylogenetic analyses have placed it outside of the mammalian V2 clade [10, 14], and the chicken receptor called VT2 [10] is in fact a homolog of the mammalian V1B, a receptor that has also been known as $V_{3}$. The cloned chicken receptors are reviewed by Baeyens and Cornett (2006) [5]. In amphibians, vasopressin (vasotocin) receptors showing similarities to mammalian V1A, V1B and V2 as well as oxytocin (mesotocin) receptors have been cloned and characterized in several species [42]. In ray-finned fishes, all described receptors had been identified as V1A receptors and oxytocin (isotocin) receptors until recently when V2-type receptors from gray bichir and medaka were cloned [27] and a V2 receptor CDNA from the Amargosa pupfish was identified [33]. Together, these findings raise questions about the evolution of the OT/VP receptor family before and during the divergence of ray-fined fishes and lobe-finned fishes (including tetrapods), and before the origin of gnathostomes. 
We initially assumed that the four mammalian receptor subtype genes might reflect the double tetraploidization early in vertebrate evolution (2R) [34, 37]. In an effort to understand the evolutionary history of this receptor family, we have collected and characterized sequences from several species representing the major gnathostome classes. It emerged that the ancestral gnathostome actually must have had at least five receptor genes and that differential losses have occurred in different evolutionary lineages. This is proven by the fact that a few lineages have retained all five of the ancestral receptor family members. Our phylogenetic analyses that clarify important aspects of the orthology-paralogy relationships also lead to a simplified terminology for the OT/VP receptor family.

\section{Methods}

\subsection{Database searches and sequence annotation}

Amino acid sequences of OT/VP receptor family members were identified using the protein family prediction feature of the Ensembl genome browser available at <www.ensembl.org $>$ [13]. The chromosome locations of the identified sequences were noted. The chromosomal locations as well as more information about the database mining, such as genome assembly versions, are available in Supplementary material 1 . To account for failures in the automated protein family predictions, sequences were also sought through TBLASTN searches [ $\underline{3}$ ] in the Ensembl database as well as in the National Center for Biotechnology Information (NCBI) Reference Sequence and trace archive databases (<http://www.ncbi.nlm.nih.gov>) using the known human V1A, V1B, V2 and OT receptor sequences as queries. Hits were considered significant if their expect values $(E)$ were lower than $\mathrm{e}^{-30}$.

All sequences in the identified Ensembl protein family were collected from the following species (names in parenthesis indicate how the different species will be referred to henceforth): Homo sapiens (human), Mus musculus (mouse), Monodelphis domestica (opossum), Ornithorhynchus anatinus (platypus), Anolis carolinensis (anole lizard), Silurana (Xenopus) tropicalis (frog), Gallus gallus (chicken), Danio rerio (zebrafish), Oryzias latipes (medaka), Gasterosteus aculeatus (stickleback) and Takifugu rubripes (fugu). The functionally characterized Ciona instestinalis (tunicate) VP receptor sequence [22] was collected and used as query in TBLASTN searches in the Ciona intestinalis genome assembly available in the Ensembl database in order to obtain its chromosomal location and reveal possibly unidentified family members. The functionally characterized Octopus vulgaris (common octopus) sequences for OPR, CTR1 and CTR2 [21] were collected to be used as an outgroup. Family members were also sought in the Callorhinchus milii (elephant shark) genome database (<http://esharkgenome.imcb.astar.edu.sg>). Genomic scaffold sequences representing the top hits (expect values $<\mathrm{e}^{-30}$ ) were collected in order to predict the receptor sequences.

All identified sequences were verified against published sequences entered into the NCBI Reference Sequence database. Erroneous predictions in the Ensembl database were replaced with better reference database sequences where available or annotated manually by following consensus for gene initiation and splice donor and acceptor sites as well as sequence similarities to verified family members. Short predictions were extended by searching for missing exons in the flanking genomic sequence. All new protein predictions were controlled for the characteristic 
seven transmembrane helices using the TMHMM prediction tool available at $<$ www.cbs.dtu.dk/services/TMHMM/> [29]. Detailed accounts of the editing process can be provided upon request.

\subsection{Sequence alignments and phylogenetic analyses}

All amino acid sequence alignments were made using the ClustalW [48] function on Jalview version 2.4.0 [52] and edited in Jalview or in the text editor TextWrangler version 3.1

(<http://www.barebones.com/products/TextWrangler>). An initial alignment was made using the identified sequences in order to create a phylogenetic tree with the neighbor joining $(\mathrm{NJ})$ method [39] (1000 bootstrap replications and standard settings in ClustalX version 2.0 [31]). The main clusters of the OT/VP receptor tree were identified in the NJ-tree topology (Supplementary material 4), after which amino acid sequence alignments for each individual cluster were made and edited manually. Poorly aligned sequence stretches were removed from the amino terminal, intracellular loop 3 (ICL3) and carboxy terminal. Alignment details are provided in

Supplementary material 3. The tunicate and octopus protein sequences were edited to remove corresponding sequence stretches. These main cluster alignments were then aligned to one another and to the tunicate and octopus protein sequences using ClustalX's profile alignment function. The final alignment was edited manually to adjust sequence stretches before and after the removed ICL3 sequence and to adjust and align conserved exon boundaries. A phylogenetic maximum likelihood (PhyML) tree was created from this alignment using the online execution of the PhyML 3.0 algorithm available at <http://www.atgc-montpellier.fr/phyml/> [15]. The amino acid frequency (equilibrium frequency), proportion of invariable sites and gamma-shape for the amino acid substitution rate heterogeneity parameters were estimated from the dataset. The number of substitution rate categories was set to 8 . The starting tree was estimated using BIONJ and both the NNI and SPR tree improvement methods were used to estimate the best tree topology. Both the tree topology and branch length optimization options were selected. A nonparametric bootstrap analysis with 100 replicates was selected for statistical branch support. The best amino acid substitution model was estimated from the final alignment using ProtTest version 1.4 [1]. In ProtTest, models were tested with no add-ons and assuming 8 gamma rate categories, the optimization strategy was set to slow and the BIONJ strategy was selected to create a random input tree. As a result of this analysis, the JTT-model of amino acid substitution was assumed for the construction of the PhyML tree.

\section{Results}

\subsection{Retrieval of receptor sequences from genome databases}

Oxytocin and vasopressin receptor protein sequences were collected from genomic databases for a variety of species representing most major gnathostome classes as well as from the urochordate tunicate Ciona intestinalis and the mollusc Octopus vulgaris in order to calculate a phylogenetic tree of the vertebrate family of OT/VP receptors. The identified protein sequences include predictions from several previously unknown OT/VP receptor genes. Not all novel predictions include the whole open reading frame (ORF), however all but 3 predictions constitute 
an uninterrupted reading frame and seven transmembrane helices could be identified for all species except elephant shark. The gene repertoires in the different species are discussed below and a summary including the number of identified exons and status of the new sequence predictions is shown in Table 1. A more comprehensive list, including chromosomal locations and database identifiers of all identified sequences is shown in Supplementary material 1. All protein sequence predictions identified in this study, together with the results of transmembrane helix predictions and additional comments, are provided in Supplementary material 2.

Many of the automatic predictions in the Ensembl database had to be revised manually in order to produce alignable protein sequence predictions. This editing work included correcting erroneous splice-site predictions and extending the coding sequence to the start and stop codons if possible. This highlights the importance of not relying on automated gene prediction and gene annotation tools when studying the evolution of gene families.

\subsection{Phylogenetic analysis}

The resulting phylogenetic maximum likelihood tree shows that the vertebrate OT/VP receptor family consists of five main clusters: V1A-type, V1B-type, the OT receptors and two main V2-type lineages that we have called V2A and V2B to mirror the naming of the V1-type receptors (Fig. 1). The distinction between V2A and V2B types has not been recognized previously. These five types are represented in tetrapod and teleost genomes as well as the genome of a holocephalan cartilaginous fish species, the elephant shark, which establishes their common gnathostome ancestry going back approx. five hundred million years [18]. A third seemingly teleost-specific V2type branch, represented by sequences in the zebrafish and stickleback genomes, has been called V2-like pending further analysis. No additional family members could be identified in the genome of the tunicate.

\subsection{OT/VP receptor genes in tetrapods}

All four previously characterized OT/VP receptor subtypes in the human genome, V1A, V1B, V2 (V2A) and OT receptors, were identified in the mouse, opossum and platypus genomes. The opossum genome assembly in Ensembl lacked a V2-type sequence and TBLASTN-searches using the human V2 receptor as query produced no significant hits. However, we could identify a receptor sequence fragment lacking only the amino terminal in two entries in the NCBI trace archive representing two exons of the V2-type gene. The chromosomal location of this opossum $\mathrm{V} 2$ receptor gene is therefore still unknown. In the chicken genome database we found the three previously reported receptor genes - V1B (known as VT2 in chicken), V2B (known as VT1) and OTR (known as MTR) [5] - as well as the fourth family member, V1A (sometimes called VT4) (Table 1). The known V2-type sequence in chicken clusters together with the teleost V2B-type sequences in our phylogenetic tree and no V2A-type sequence could be identified in the chicken genome or in any of the other available avian genomes, namely those of the zebra finch (Taeniopygia guttata), turkey (Meleagris gallopavo) and mallard (Anas platyrhynchos) (data not shown). Both V2A-type and V2B-type receptor subtypes were retrieved from the frog and anole lizard genome databases in addition to the V1A, V1B and OT receptors. These two species represents the only tetrapods in this study that have retained all five ancestral lineages. 


\section{Table 1}

Summary of OT/VP receptor sequences analyzed in this study.

\begin{tabular}{|c|c|c|c|c|c|c|c|c|c|}
\hline & $\begin{array}{l}\text { Genus and species } \\
\text { (common name) }\end{array}$ & $\begin{array}{l}\text { Gene } \\
\text { name }^{\text {a }}\end{array}$ & $\begin{array}{l}\text { Protein } \\
\text { name }^{\text {a }}\end{array}$ & Subtype & $\begin{array}{l}\text { Sequence } \\
\text { length }^{\mathrm{b}}\end{array}$ & $\begin{array}{l}\text { No. of } \\
\text { exons }\end{array}$ & $\begin{array}{l}\text { TM regions in } \\
\text { new } \\
\text { predictions }\end{array}$ & $\begin{array}{l}\text { Status of } \\
\text { new } \\
\text { predictions }\end{array}$ & References $^{\mathrm{g}}$ \\
\hline \multirow[t]{16}{*}{ Mammals } & \multirow{4}{*}{$\begin{array}{l}\text { Homo sapiens } \\
\text { (human) }\end{array}$} & AVPR1A & $V_{1 A}$ & V1A-type & $419(297)$ & 2 & & & {$[\underline{46}, \underline{47}]$} \\
\hline & & $A V P R 1 B$ & $V_{1 B}$ & V1B-type & $425(298)$ & 2 & & & {$[\underline{38}, \underline{44}]$} \\
\hline & & AVPR2 & $V_{2}$ & V2A-type & $372(293)$ & 3 & & & {$[\underline{7}, \underline{43}]$} \\
\hline & & OXTR & OT & OTR-type & $390(293)$ & 2 & & & {$[\underline{19}, \underline{24}]$} \\
\hline & \multirow{4}{*}{$\begin{array}{l}\text { Mus musculus } \\
\text { (mouse) }\end{array}$} & Avpr1a & $V_{1 A}$ & V1A-type & $424(297)$ & 2 & & & [23] \\
\hline & & Avprib & $V_{1 B}$ & V1B-type & $422(298)$ & 2 & & & {$[\underline{23}, \underline{50}]$} \\
\hline & & Avpr2 & $V_{2}$ & V2A-type & $372(293)$ & 3 & & & [35] \\
\hline & & Oxtr & OT & OTR-type & $389(293)$ & & & & [30] \\
\hline & \multirow{4}{*}{$\begin{array}{l}\text { Monodelphis } \\
\text { domestica } \\
\text { (opossum) }\end{array}$} & & & V1A-type & $421(297)$ & 2 & 7 & Whole ORF & $\mathrm{g}$ \\
\hline & & & & V1B-type & $421(298)$ & 2 & 7 & Whole ORF & $\mathrm{g}$ \\
\hline & & & & V2A-type & $356(289)$ & 2 & 7 & c & $\mathrm{g}$ \\
\hline & & & & OTR-type & $398(293)$ & 2 & 7 & Whole ORF & $\mathrm{g}$ \\
\hline & \multirow{4}{*}{$\begin{array}{l}\text { Ornithorynchus } \\
\text { anatinus (platypus) }\end{array}$} & & & V1A-type & $429(297)$ & 2 & 7 & Whole ORF & $\mathrm{g}$ \\
\hline & & & & V1B-type & $266(187)$ & 2 & 4 (TM4-TM7) & d & $\mathrm{g}$ \\
\hline & & & & V2A-type & $382(285)$ & 2 & 7 & Whole ORF & $\mathrm{g}$ \\
\hline & & & & OTR-type & $393(293)$ & 2 & 7 & Whole ORF & $\mathrm{g}$ \\
\hline \multirow[t]{4}{*}{ Birds } & \multirow{4}{*}{$\begin{array}{l}\text { Gallus gallus } \\
\text { (chicken) }\end{array}$} & AVPR1A & VT4 & V1A-type & $420(297)$ & 2 & 7 & Whole ORF & $\mathrm{g}$ \\
\hline & & AVPR1B & VT2 & V1B-type & $426(298)$ & 2 & & & {$[\underline{10}]$} \\
\hline & & AVPR2 & VT1 & V2B-type & $371(297)$ & 2 & & & [45] \\
\hline & & OXTR & MTR & OTR-type & $392(293)$ & 2 & & & [14] \\
\hline \multirow[t]{5}{*}{ Reptiles } & \multirow{5}{*}{$\begin{array}{l}\text { Anolis carolinensis } \\
\text { (anole lizard) }\end{array}$} & & & V1A-type & $409(298)$ & 2 & 7 & Whole ORF & $\mathrm{g}$ \\
\hline & & & & V1B-type & $423(298)$ & 2 & 7 & Whole ORF & $\mathrm{g}$ \\
\hline & & & & V2A-type & $333(297)$ & 2 & 7 & c & $\mathrm{g}$ \\
\hline & & & & V2B-type & $335(283)$ & 2 & 7 & c & $\mathrm{g}$ \\
\hline & & & & OTR-type & $390(293)$ & 2 & 7 & Whole ORF & $\mathrm{g}$ \\
\hline \multirow[t]{5}{*}{ Amphibians } & \multirow{5}{*}{$\begin{array}{l}\text { Silurana (Xenopus) } \\
\text { tropicalis (frog) }\end{array}$} & & & V1A-type & $412(296)$ & 2 & 7 & Whole ORF & $\mathrm{g}$ \\
\hline & & & & V1B-type & $393(298)$ & 2 & 7 & Whole ORF & $\mathrm{g}$ \\
\hline & & & & V2A-type & $362(285)$ & 2 & 7 & Whole ORF & $\mathrm{g}$ \\
\hline & & & & V2B-type & $346(292)$ & 2 & 7 & Whole ORF & $\mathrm{g}$ \\
\hline & & & & OTR-type & $391(293)$ & 2 & 7 & Whole ORF & $\mathrm{g}$ \\
\hline \multirow[t]{21}{*}{ Teleost fish } & \multirow{8}{*}{$\begin{array}{l}\text { Danio rerio } \\
\text { (zebrafish) }\end{array}$} & & & V1A-type (1) & $411(297)$ & 2 & 7 & Whole ORF & $\mathrm{g}$ \\
\hline & & & & V1A-type (2) & $380(296)$ & 2 & 7 & Whole ORF & $\mathrm{g}$ \\
\hline & & & & V2A-type (1) & $543(288)$ & 5 & 7 & Whole ORF & $\mathrm{g}$ \\
\hline & & & & V2A-type (2) & $531(288)$ & 5 & 7 & Whole ORF & $\mathrm{g}$ \\
\hline & & & & V2B-type & $362(292)$ & 2 & 7 & Whole ORF & $\mathrm{g}$ \\
\hline & & & & OTR-type (1) & $383(293)$ & 2 & 7 & Whole ORF & $\mathrm{g}$ \\
\hline & & & & OTR-type (2) & $389(288)$ & 2 & 7 & Whole ORF & $\mathrm{g}$ \\
\hline & & & & V2-like & $309(257)$ & 2 & 7 & Whole ORF & $\mathrm{g}$ \\
\hline & \multirow{7}{*}{$\begin{array}{l}\text { Oryzias latipes } \\
\text { (medaka) }\end{array}$} & & & V1A-type (1) & $380(297)$ & 2 & 7 & Whole ORF & $\mathrm{g}$ \\
\hline & & & & V1A-type (2) & $411(297)$ & 2 & 7 & Whole ORF & $\mathrm{g}$ \\
\hline & & & $\mathrm{V} 2 \mathrm{R}$ & V2A-type (1) & $543(288)$ & 5 & & & [27] \\
\hline & & & & V2A-type (2) & $531(288)$ & 5 & 7 & ce & $\mathrm{g}$ \\
\hline & & & & V2B-type & $362(292)$ & 2 & 7 & c & $\mathrm{g}$ \\
\hline & & & & OTR-type (1) & $389(293)$ & 2 & 7 & Whole ORF & $\mathrm{g}$ \\
\hline & & & & OTR-type (2) & $383(288)$ & 2 & 7 & Whole ORF & $\mathrm{g}$ \\
\hline & \multirow{6}{*}{$\begin{array}{l}\text { Gasterosteus } \\
\text { aculeatus } \\
\text { (stickleback) }\end{array}$} & & & V1A-type (1) & $387(297)$ & 2 & 7 & Whole ORF & $\mathrm{g}$ \\
\hline & & & & V1A-type (2) & $388(293)$ & 2 & 7 & Whole ORF & $\mathrm{g}$ \\
\hline & & & & V2A-type (1) & 356 (289) & 5 & 7 & ce & $\mathrm{g}$ \\
\hline & & & & V2A-type (2) & $396(287)$ & 5 & 7 & c & $\mathrm{g}$ \\
\hline & & & & V2B-type & $335(292)$ & 2 & 7 & c & $\mathrm{g}$ \\
\hline & & & & OTR-type & $393(296)$ & 2 & 7 & Whole ORF & $\mathrm{g}$ \\
\hline
\end{tabular}




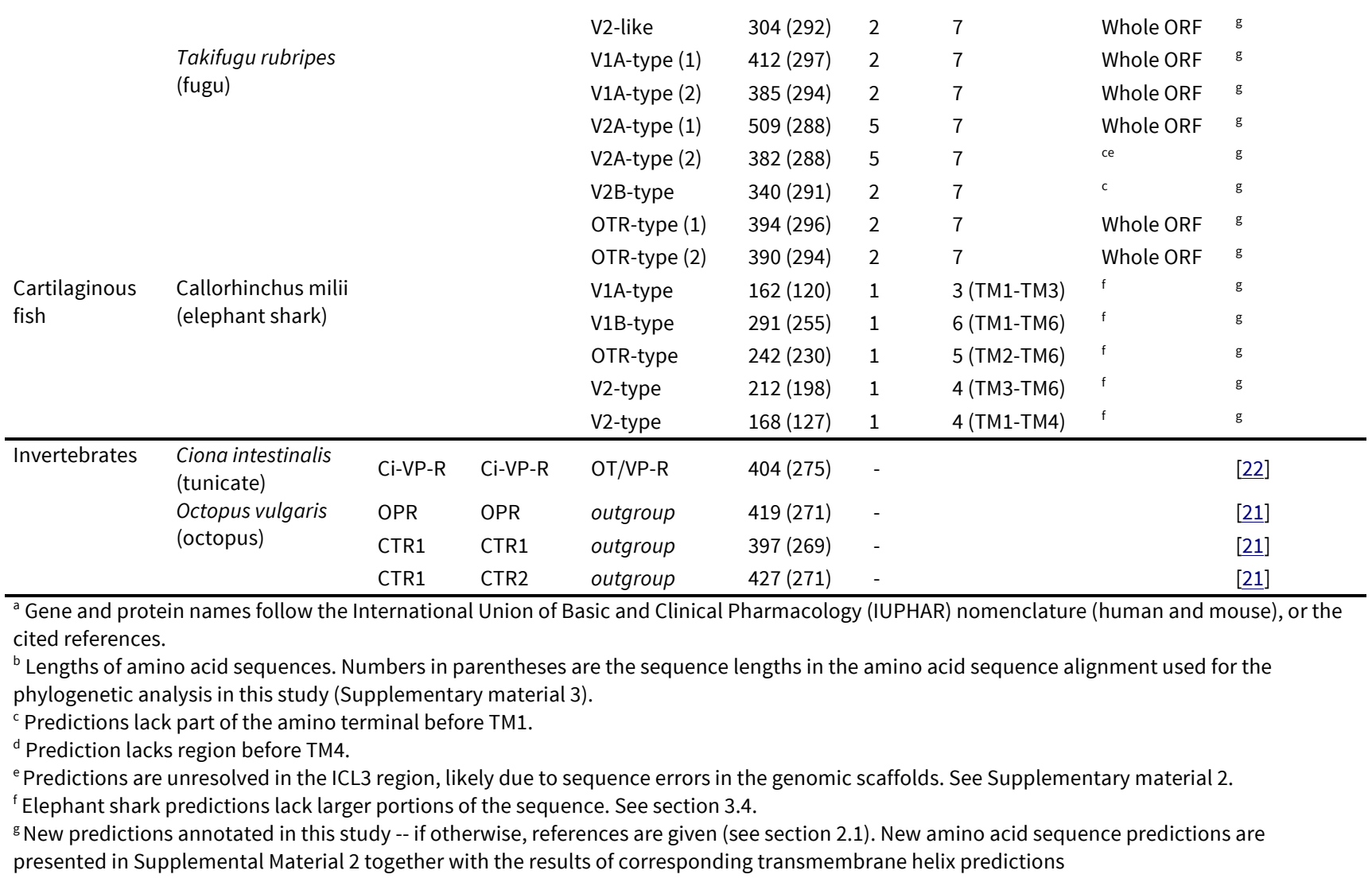

\subsection{OT/VP receptor genes in elephant shark}

The TBLASTN-searches in the holocephalan elasmobranch elephant shark genome produced hits on five genomic scaffolds with expect values lower than $\mathrm{e}^{\wedge}-30$. From these scaffold sequences, five receptor protein sequence fragments were predicted using TMHMM transmembrane helix predictions and sequence similarities to the human OT/VP receptors. Scaffold IDs can be seen in Fig. 1 and Supplementary material 1 and full scaffold sequences can be retrieved from <http://blast.fugu-sg.org/cgi-bin/scripts/request_scaff.pl>. These elephant shark OT/VP receptor fragments establish the V1A-type, V1B-type and OT receptors as ancestral gnathostome receptor types. The two V2-type receptor fragments cluster with V2B-type receptors (Fig. 1, Supplementary material 4) and may represent an independent duplication within the cartilaginous fish lineage. The identified fragments constitute open reading frames of a single exon and span between 162 and 291 amino acid residues (Table 1).

\subsection{OT/VP receptor genes in teleosts}

All teleost genomes included in the analysis were found to have multiple V1A-type and V2A-type receptor sequences (Table 1, Fig. 1). All sequenced teleost genomes except the stickleback genome also have OT receptor duplicates. It was not possible to identify any V1B-type receptor genes in the teleost genomes by our sequence searches and phylogenetic analyses. The V2A-type duplicates seen in the teleost genomes are likely to have arisen by tandem duplication since the two copies are located adjacent to each other in all analyzed teleost genome assemblies (Fig. 1, 
see Supplementary material 1 for detailed chromosomal locations). This is the same case with the OT-type duplicates in those teleost genomes where they are found. The duplicate V1A-type receptors are located on different chromosomes in the zebrafish and medaka genomes, and in the stickleback genome only one has been assigned to a chromosome, suggesting that they arose by chromosome duplication. However, the phylogeny for these clusters is not resolved for the zebrafish (Fig. 1, Supplementary material 4) and it is difficult to say whether the duplicates in teleosts represent the same or independent events in all species. The fugu genomic scaffolds have not yet been mapped to chromosomes which provides no useful information in this regard.

Regarding the exon-intron organization for V2A, we identified three unique introns in all teleost V2A genes, all located between a CAG and a GTG/T codon. Another distinctive feature of V2A in teleosts is the considerable expansion of intracellular loop three (ICL3). These features complicated the manual annotation of the amino acid sequences, which in several cases were found to be erroneous or incomplete in the automatic Ensembl database gene predictions.

Fig. 1. Phylogenetic maximum likelihood tree of the oxytocin and vasopressin receptor family showing five main gnathostome branches. Species abbreviations: Aca (anole lizard), Dre (zebrafish), Cin (tunicate), Cmi (elephant shark), Gac (stickleback), Gga (chicken), Hsa (human), Mdo (opossum), Mmu (mouse), Oan (platypus), Ola (medaka), Ovu (octopus), Tru (fugu), Xtr (frog). Where known, the chromosome assignments of the identified receptor sequences are given next to the sequence name. For the elephant shark sequences, the scaffold IDs are shown (see section 3.4). Sequences marked with an asterisk are fragments and in some cases do not span the entire length of the sequence alignment used to construct this tree (see Table 1 and Supplementary material 3). Branch support is shown for each node in percent of 100 bootstrap iterations. The nodes of the five main branches are well supported, with bootstrap values of $82 \%$ for the V2B-branch, 94\% for the V1A-branch and 100\% for the V2A-, V1B- and OTR-branches. Nodes with bootstrap support lower than $50 \%$ are marked with red arrowheads and those with support lower than $75 \%$ are marked with yellow arrowheads.

\section{Discussion}

The phylogenetic tree presented here (Fig. 1) shows that the oxytocin and vasopressin receptor family consists of at least five lineages or subtypes in the gnathostome lineage, as evidenced by the presence of all subtypes in the anole lizard, frog and teleost fish genomes (Fig. 2). In addition to the previously recognized subtypes $\mathrm{V} 1 \mathrm{~A}, \mathrm{~V} 1 \mathrm{~B}, \mathrm{~V} 2$ and OTR, we have found that there is a distinct V2 subtype both in tetrapods and teleost fishes that we have named V2B; hence we call the $\mathrm{V} 2$ receptor subtype previously established in mammals V2A. To the best of our knowledge, this is the first comprehensive phylogenetic study of this family using extensive searches of the available genomic sequence resources and multiple phylogenetic methods. It also constitutes the first description of a whole V2-type receptor lineage, including at least two major subtypes, V2A and V2B, as well as lineage-specific V2 duplicates in several species of teleost fishes. All teleost genomes display duplicate copies of V2A-type receptors. Interestingly, such duplication indicates that some teleost fish species may possess as many as 4 different V2-type receptors (Table 1, Fig. 1 and Fig. 2). Functional expression of these receptors in cell lines will be the next step towards characterization of their peptide-binding preferences and signal transduction pathways. Recently a V2-type sequence was reported and described in the Amargosa pupfish 
Cyprinodon nevadensis amargosa [33], as well as in medaka and the gray bichir Polypterus senegalus [27]. Our analyses (Supplementary material 5) identify the pupfish V2 receptor as a $\mathrm{V} 2 \mathrm{~A}(1)$ receptor, the gray bichir $\mathrm{V} 2$ receptor as a $\mathrm{V} 2 \mathrm{~A}$ receptor clustering basally to teleost $\mathrm{V} 2 \mathrm{~A}(1)$ and $\mathrm{V} 2 \mathrm{~A}(2)$ receptors and the medaka receptor as the $\mathrm{V} 2 \mathrm{~A}(1)$ on chromosome 7 (Fig. 1), one of three different V2-type receptor sequences identified by us in the genome database of this species.

Fig. 2. Proposed evolution of the oxytocin and vasopressin receptor family in gnathostomes. The upper panel shows a cladogram of the phylogenetic maximum likelihood analysis presented in Fig. 1 and the left side panel shows a timetree of chordates. Arrowhead numbers: 1) Expansion of the OT/VP-R family early in vertebrate evolution. 2) Loss of V1B-type receptors, duplications of V2A-, OT- and V1A-receptors before the teleost divergence. Likely emergence of the V2-like gene, with subsequent losses in the medaka and fugu genomes. 3) Loss of V2A-receptors in the avian lineage. 4) Loss of V2B-receptors in the mammalian lineage. The two elephant shark V2-type sequences have been colored grey because of ambiguous orthology (see Section 3.4).

The V1A-, V1B- and OT-type receptor clusters are clearly rooted in the gnathostome ancestry by homologous elephant shark receptor sequence fragments (see section 3.5 above). However, the topology is less clear regarding the V2-type lineages. The node separating the V2Atype and V2B-type lineages has sufficient branch support in the phylogenetic tree, but it is not clear to which lineage each of the two elephant shark V2-type receptor sequence fragments belongs. Because they both cluster with V2B sequences (Fig. 1), they might represent an independent duplication within the cartilaginous fish lineage. However, their placement in the tree is likely to be compromised by the relative shortness of the identified sequence fragments relative to the alignment (Supplementary material 3). For definitive assignment to specific receptor types, complete sequences will be necessary. In any case, the presence of two different V2-type receptor sequences in the tetrapods anole lizard and frog (see Section 3.3 above, as well as Fig. 1), and in teleosts (see Section 3.5 above) confirms that both lineages were present at the divergence of lobe-finned and ray-finned fishes. The presence of all five OT/VP receptor lineages as far back as the gnathostome ancestor, approx. five hundred million years ago [18], is suggested by the presence of two V2-type receptor fragments in the elephant shark genome sequence.

After the divergence of cartilaginous fishes the receptor family appears to have undergone independent gains and losses in different lineages. The phylogenetic tree shows that the chicken V2-type receptor (unfortunately called VT1 in its first publication) [45] is not orthologous to the mammalian V2-type receptor but that they represent distinct ancestral gnathostome lineages, the V2B-type and V2A-type respectively (Fig. 1). Thus, it appears as though there have been lineage specific reciprocal losses in the bird and mammal lineages. This is corroborated by the fact that out of the tetrapod genome sequences included in this study, only the frog and anole lizard genomes possess all five ancestral subtypes. Avian V2A-type sequences were sought using TBLASTN with the human V2A-type sequence as query in the other avian genome sequences available in the Ensembl database (see Section 3.3 above), however this produced no significant hits. In contrast to the mammalian V2A receptors, the chicken V2B receptor does not mediate an increase in intracellular cAMP, rather it seems to signal via the phosphatidylinositol pathway, increasing cytosolic $\mathrm{Ca}^{2+}$ much like mammalian V1A, V1B and OT receptors [45]. This raises the question of whether CAMP signaling is specific to receptors of the V2A subtype and it will be 
interesting to see whether reptile, amphibian and teleost $\mathrm{V} 2 \mathrm{~B}$ receptors signal through the same pathway as the chicken V2B.

In the teleost fishes various duplications and losses have occurred. Notably, no V1B-type sequence has been found in a teleost. A putative V1B ortholog was found in the elephant shark, suggesting that V1B was lost in the teleost ancestor or even earlier in the ray-finned fish lineage. Because we have found all five ancestral subtypes in the genomes of a frog and a lizard, it is reasonable to expect that lungfishes and coelacanths may still possess a V1B-type ortholog as well, although to date only a V2-type receptor and one V1-type receptor have been identified in the African lungfish Protopterus annectens [25, 26].

The fact that multiple independent duplication events have shaped the receptor family in teleosts highlights the importance of determining the chromosomal locations in order to deduce the correct orthology relationships. The same would apply to identified family members in other teleost species whose genomes have not been sequenced. The chromosomal locations of the duplicate OT-type and V2A-type receptor genes in zebrafish, medaka and fugu suggest ancestral teleost tandem duplications. The same scenario is suggested by the chromosomal locations of the V1A duplicates in the zebrafish genome, while the stickleback and medaka genome assemblies suggest that independent duplication events may have occurred. A more extensive analysis of paralogous chromosome regions is in progress to see how the OT/VP receptor gene duplications may correlate with whole genome duplications, however this work is exceedingly complicated due to extensive rearrangements of (some of) these chromosomes during vertebrate evolution.

The human V1A, V1B and OT receptors are encoded by two exons while the human V2 receptor (V2A) is encoded by three [43]. The first intron of the human V2A receptor gene is suggested to be a mammal-specific insertion while the second intron is an ancestral intron found in all vertebrate OT/VP receptors [8]. Our findings support this view. Although the complete amino terminal was not possible to predict with certainty for all newly identified V2A sequences, such as the opossum V2A or several of the teleost V2As, it is present in the platypus V2A gene prediction and absent in the teleost V2A sequences where the complete amino terminal could be identified (Table 1 ). Interestingly, our predictions of the teleost V2A-type receptor genes reveal three introns specific for this subtype in this lineage (in addition to the ancestral vertebrate intron). All three introns appear to have been inserted in the same sequence motif, CAG GTG/T (see alignment in Supplementary material 3), and the splice donor and acceptor sites have remained almost identical in all teleost genomes investigated in this study (data not shown). Surprisingly, the putative Armargosa pupfish V2-type receptor published by Lema (2010) [33] features only the last of these three introns and not the ancestral intron homologous to the second intron in the human $\mathrm{V} 2$ receptor gene. This deviating exon-intron organization in the pupfish requires further investigation. Konno et al (2010) [27] did not investigate the genomic DNA sequences of gray bichir and medaka.

Another distinctive feature of the teleost V2A-type receptors is the significant elongation of the middle portion of intracellular loop three (ICL3). This elongation could be identified in all teleost V2A sequences, while this region of the medaka V2A(2), stickleback V2A(1) and fugu V2A(2) sequences could not be correctly predicted due to interrupted reading frames and the lack of canonical splice sites in this region of the genomic sequence available in the databases (Table 1, see sequences in Supplementary material 2). This is likely due to faulty sequence reads in the genomic scaffolds. Our predictions of the ICL3 expansion in the teleost V2A-type receptor sequences included in this analysis are supported by the identification of corresponding 
expansions in the Amargosa pupfish and medaka V2 sequences published by Lema (2010) and Konno et al. (2010), respectively. Interestingly Konno et al. (2010) report that the gray bichir V2 receptor sequence does not present the ICL3 elongation, which suggests that his feature appeared early in teleost evolution, before the teleost radiation [27]. ICL3 displays high variability in both length and amino acid composition in mammalian V2 receptors and the relatively low functional activity of the middle part of the loop in the human $\mathrm{V} 2$ receptor has been demonstrated [ $\underline{36}, \underline{41}]$.

Apart from the suggested five ancestral gnathostome lineages, a sixth lineage, representing a V2-subtype, is present in the zebrafish and stickleback genomes. We have called this subtype V2-like pending further evolutionary analysis. Since no member of this lineage was found in any other genomes, its orthology relationship remains unknown. Both sequences seem upon inspection to be able to give rise to functional receptors. However, the carboxy terminal has been significantly shortened by the introduction of a stop codon (see sequences in Supplementary materials 2 and 3 ).

\section{Conclusions}

Our investigation of OT/VP receptors in the available genome sequences of species representing different gnathostome classes allowed us to deduce a phylogenetic tree that provided some surprising findings. We conclude that there were at least five receptor subtypes present already in the gnathostome ancestor; V1A, V1B, V2A, V2B and OT receptors, thus a larger receptor repertoire in the vertebrate lineage than was previously known. Secondly, reciprocal losses seem to have occurred in some lineages, namely V2B from mammals and V2A from birds. Comparative studies of the neurohypophysial hormones have for a long time been muddled by an inconsistent and evolutionarily misleading nomenclature. Our comprehensive phylogenetic analysis of the OT/VP receptors resolves orthology relationships and corrects previous cases of subtype misidentification. These evolutionary clarifications, together with the resolution of oxytocin and vasopressin peptide evolution $[\underline{16}, \underline{17}]$, provide a more coherent framework for comparative studies of the many functions of neurohypophysial hormones and their receptors.

\section{Role of the funding source}

This work was supported by grants from The Swedish Research Council and Carl Trygger's Foundation. These funding bodies had no role in the study design; in the collection, analysis, and interpretation of data; in the writing of the report; or in the decision to submit the paper for publication.

\section{Acknowledgements}

We thank Dr. Tomas A. Larsson (presently at the EMBL, Heidelberg, Germany) and Pasi Bauer who performed the initial preliminary analyses, Dr. Görel Sundström for stimulating discussions and Dr. Venkatesh (Institute of Molecular and Cell Biology, Singapore) for the discussion about the unsolved prediction of intracellular loop 3 (IL3) in fugu V2A2. 


\section{Supplementary data}

Supplementary data associated with this article can be found from the original publisher, in the online version, at doi:10.1016/j.ygcen.2011.10.011. All supplementary data have also been deposited for free access along with this article on figshare at doi:10.6084/m9.figshare.811860.

\section{References}

[1] F. Abascal, R. Zardoya, D. Posada, ProtTest: selection of best-fit models of protein evolution, Bioinformatics 21 (2005) 2104-2105.

[2] R. Acher, Molecular evolution of biologically active polypeptides, Proc. Biol. Sci. 210 (1980) 2143.

[3] S.F. Altschul, W. Gish, W. Miller, E.W. Myers, D.J. Lipman, Basic local alignment search tool, J. Mol. Biol. 215 (1990) 403-410.

[4] T. Backström, S. Winberg, Arginine-vasotocin influence on aggressive behavior and dominance in rainbow trout, Physiol. Behav. 96 (2009) 470-475.

[5] D.A. Baeyens, L.E. Cornett, The cloned avian neurohypophysial hormone receptors, Comp. Biochem. Physiol. B, Biochem. Mol. Biol. 143 (2006) 12-19.

[6] R.J. Balment, W. Lu, E. Weybourne, J.M. Warne, Arginine vasotocin a key hormone in fish physiology and behaviour: a review with insights from mammalian models, Gen. Comp. Endocrinol. 147 (2006) 9-16.

[7] M. Birnbaumer, A. Seibold, S. Gilbert, M. Ishido, C. Barberis, A. Antaramian, P. Brabet, W. Rosenthal, Molecular cloning of the receptor for human antidiuretic hormone, Nature 357 (1992) 333-335.

[8] I. Böselt, H. Römpler, T. Hermsdorf, D. Thor, W. Busch, A. Schulz, T. Schöneberg, Involvement of the V2 vasopressin receptor in adaptation to limited water supply, PLoS ONE 4 (2009) e5573.

[9] C.W. Bourque, Central mechanisms of osmosensation and systemic osmoregulation, Nat. Rev. Neurosci. 9 (2008) 519-531.

[10] L.E. Cornett, J.D. Kirby, J.A. Vizcarra, J.C. Ellison, J. Thrash, P.R. Mayeux, M.D. Crew, S.M. Jones, N. Ali, D.A. Baeyens, Molecular cloning and functional characterization of a vasotocin receptor subtype expressed in the pituitary gland of the domestic chicken (Gallus domesticus): avian homolog of the mammalian V1b-vasopressin receptor, Regul. Pept. 110 (2003) 231-239.

[11] Z.R. Donaldson, L.J. Young, Oxytocin, vasopressin, and the neurogenetics of sociality, Science 322 (2008) 900-904.

[12] J.N. Ferguson, L.J. Young, E.F. Hearn, M.M. Matzuk, T.R. Insel, J.T. Winslow, Social amnesia in 
mice lacking the oxytocin gene, Nat. Genet. 25 (2000) 284-288.

[13] P. Flicek, M.R. Amode, D. Barrell, K. Beal, S. Brent, Y. Chen, P. Clapham, G. Coates, S. Fairley, S. Fitzgerald, L. Gordon, M. Hendrix, T. Hourlier, N. Johnson, A. Kähäri, D. Keefe, S. Keenan, R. Kinsella, F. Kokocinski, E. Kulesha, P. Larsson, I. Longden, W. McLaren, B. Overduin, B. Pritchard, H.S. Riat, D. Rios, G.R.S. Ritchie, M. Ruffier, M. Schuster, D. Sobral, G. Spudich, Y.A. Tang, S. Trevanion, J. Vandrovcova, A.J. Vilella, S. White, S.P. Wilder, A. Zadissa, J. Zamora, B.L. Aken, E. Birney, F. Cunningham, I. Dunham, R. Durbin, X.M. Fernández-Suarez, J. Herrero, T.J.P. Hubbard, A. Parker, G. Proctor, J. Vogel, S.M.J. Searle, Ensembl 2011, Nucleic Acids Res. 39 (2011) D800-806.

[14] K.I. Gubrij, C.M. Chaturvedi, N. Ali, L.E. Cornett, J.D. Kirby, J. Wilkerson, M. Mikhailova, M.L. Turner, D.A. Baeyens, Molecular cloning of an oxytocin-like receptor expressed in the chicken shell gland, Comp. Biochem. Physiol. B, Biochem. Mol. Biol. 142 (2005) 37-45.

[15] S. Guindon, O. Gascuel, A simple, fast, and accurate algorithm to estimate large phylogenies by maximum likelihood, Syst. Biol. 52 (2003) 696-704.

[16] P.-C. Gwee, C.T. Amemiya, S. Brenner, B. Venkatesh, Sequence and organization of coelacanth neurohypophysial hormone genes: evolutionary history of the vertebrate neurohypophysial hormone gene locus, BMC Evol. Biol. 12 (2008) 1-12.

[17] P.-C. Gwee, B.-h. Tay, S. Brenner, B. Venkatesh, Characterization of the neurohypophysial hormone gene loci in elephant shark and the Japanese lamprey: origin of the vertebrate neurohypophysial hormone genes, BMC Evol. Biol. 15 (2009) 1-15.

[18] B.M. Hallström, A. Janke, Gnathostome phylogenomics utilizing lungfish EST sequences, Mol. Biol. Evol. 26 (2009) 463-471.

[19] T. Inoue, T. Kimura, C. Azuma, J. Inazawa, M. Takemura, T. Kikuchi, Y. Kubota, K. Ogita, F. Saji, Structural organization of the human oxytocin receptor gene, J. Biol. Chem. 269 (1994) 32451-32456.

[20] T.R. Insel, The challenge of translation in social neuroscience: a review of oxytocin, vasopressin, and affiliative behavior, Neuron 65 (2010) 768-779.

[21] A. Kanda, H. Satake, T. Kawada, H. Minakata, Novel evolutionary lineages of the invertebrate oxytocin/vasopressin superfamily peptides and their receptors in the common octopus (Octopus vulgaris), Biochem. J. 387 (2005) 85-91.

[22] T. Kawada, T. Sekiguchi, Y. Itoh, M. Ogasawara, H. Satake, Characterization of a novel vasopressin/oxytocin superfamily peptide and its receptor from an ascidian, Ciona intestinalis, Peptides 29 (2008) 1672-1678.

[23] S. Kikuchi, A. Tanoue, N. Goda, N. Matsuo, G. Tsujimoto, Structure and sequence of the mouse V1a and V1b vasopressin receptor genes, Jpn. J. Phamacol. 81 (1999) 388-392. 
[24] T. Kimura, O. Tanizawa, K. Mori, M.J. Brownstein, H. Okayama, Stucture and expression of a human oxytocin receptor, Nature 356 (1992) 526-529.

[25] N. Konno, S. Hyodo, Y. Yamaguchi, H. Kaiya, M. Miyazato, K. Matsuda, M. Uchiyama, African lungfish, Protopterus annectens, possess an arginine vasotocin receptor homologous to the tetrapod V2-type receptor, J. Exp. Biol. 212 (2009) 2183-2193.

[26] N. Konno, S. Hyodo, Y. Yamaguchi, K. Matsuda, M. Uchiyama, Vasotocin/V2-type receptor/aquaporin axis exists in African lungfish kidney but is functional only in terrestrial condition, Endocrinol. 151 (2010) 1089-1096.

[27] N. Konno, M. Kurosawa, H. Kaiya, M. Miyazato, K. Matsuda, M. Uchiyama, Molecular cloning and characterization of V2-type receptor in two ray-finned fish, gray bichir, Polypterus senegalus and medaka, Oryzias latipes, Peptides (2010) 1-7.

[28] M. Kosfeld, M. Heinrichs, P.J. Zak, U. Fischbacher, E. Fehr, Oxytocin increases trust in humans, Nature 435 (2005) 673-676.

[29] A. Krogh, B. Larsson, G. von Heijne, E.L. Sonnhammer, Predicting transmembrane protein topology with a hidden Markov model: application to complete genomes, J. Mol. Biol. 305 (2001) 567-580.

[30] Y. Kubota, T. Kimura, K. Hashimoto, Y. Tokugawa, K. Nobunaga, C. Azuma, F. Saji, Y. Murata, Structure and expression of the mouse oxytocin receptor gene, Mol. Cell. Endocrinol. 124 (1996) 25-32.

[31] M.A. Larkin, G. Blackshields, N.P. Brown, R. Chenna, P.A. McGettigan, H. McWilliam, F. Valentin, I.M. Wallace, A. Wilm, R. Lopez, J.D. Thompson, T.J. Gibson, D.G. Higgins, Clustal W and Clustal X version 2.0, Bioinformatics 23 (2007) 2947-2948.

[32] H.-J. Lee, A.H. Macbeth, J.H. Pagani, W.S. Young, Oxytocin: the great facilitator of life, Prog. Neurobiol. 88 (2009) 127-151.

[33] S.C. Lema, Identification of multiple vasotocin receptor cDNAs in teleost fish: sequences, phylogenetic analysis, sites of expression, and regulation in the hypothalamus and gill in response to hyperosmotic challenge, Mol. Cell. Endocrinol. 321 (2010) 215-230.

[34] Y. Nakatani, H. Takeda, Y. Kohara, S. Morishita, Reconstruction of the vertebrate ancestral genome reveals dynamic genome reorganization in early vertebrates, Genome Res. 17 (2007) 1254-1265.

[35] A. Oksche, G. Leder, S. Valet, M. Platzer, K. Hasse, S. Geist, G. Krause, A. Rosenthal, W. Rosenthal, Variant amino acids in the extracellular loops of murine and human vasopressin $\mathrm{V} 2$ receptors account for differences in cell surface expression and ligand affinity, Mol. Endocrinol. 16 (2002) 799-813. 
[36] Y. Pan, P. Wilson, J. Gitschier, The effect of eight V2 vasopressin receptor mutations on stimulation of adenylyl cyclase and binding to vasopressin, J. Biol. Chem. (1994) 2693193326931937.

[37] N. Putnam, T. Butts, D. Ferrier, R. Furlong, U. Hellsten, T. Kawashima, M. Robinson-Rechavi, E. Shoguchi, A. Terry, J. Yu, E. Benito-Gutiérrez, I. Dubchak, J. Garcia-Fernàndez, J.J. GibsonBrown, I.V. Grigoriev, A.C. Horton, P.J. de Jong, J. Jurka, V.V. Kapitonov, Y. Kohara, Y. Kuroki, E. Lidquist, S. Lucas, K. Osoegawa, L.A. Pennacchio, A.A. Salamov, Y. Satou, T. Sauka-Spengler, J. Schutz, T. Shin-I, A. Toyoda, M. Bronner-Fraser, A. Fujiyama, L.Z. Holland, P.W.H. Holland, N. Satoh, D.S. Rokshar, The amphioxus genome and the evolution of the chordate karyotype, Nature 453 (2008) 1064-1072.

[38] P. René, F. Lenne, M.a. Ventura, X. Bertagna, Y. de Keyzer, Nucleotide sequence and structural organization of the human vasopressin pituitary receptor (V3) gene, Gene 241 (2000) 57-64.

[39] N. Saitou, M. Nei, The neighbor-joining method: a new method for reconstructing phylogenetic trees, Mol. Biol. Evol. 4 (1987) 406-425.

[40] N. Santangelo, A.H. Bass, Individual behavioral and neuronal phenotypes for arginine vasotocin mediated courtship and aggression in a territorial teleost, Brain Behav. Evol. 75 (2010) 282-291.

[41] T. Schoneberg, J. Yun, D. Wenkert, J. Wess, Functional rescue of mutant V2 vasopressin receptors causing nephrogenic diabetes insipidus by a co-expressed receptor polypeptide, EMBO J. 15 (1996) 1283-1291.

[42] B.T. Searcy, C.S. Bradford, R.R. Thompson, T.M. Filtz, F.L. Moore, Identification and characterization of mesotocin and V1a-like vasotocin receptors in a urodele amphibian, Taricha granulosa, Gen. Comp. Endocrinol. 170 (2011) 131-143.

[43] A. Seibold, P. Brabet, W. Rosenthal, M. Birnbaumer, Structure and chromosomal localization of the human antidiuretic hormone receptor gene, Am. J. Hum. Genet. 51 (1992) 1078-1083.

[44] T. Sugimoto, M. Saito, S. Mochizuki, Y. Watanabe, S. Hashimoto, H. Kawashima, Molecular cloning and functional expression of a cDNA encoding the human V1b vasopressin receptor, J. Biol. Chem. 269 (1994) 27088-27092.

[45] F.L. Tan, S.J. Lolait, M.J. Brownstein, N. Saito, V. Macleod, D.A. Baeyens, P.R. Mayeux, S.M. Jones, L.E. Cornett, Molecular cloning and functional characterization of a vasotocin receptor subtype that is expressed in the shell gland and brain of the domestic chicken, Biol. Reprod. 62 (2000) 8-15.

[46] M. Thibonnier, C. Auzan, Z. Madhun, P. Wilkins, L. Berti-Mattera, E. Clauser, Molecular cloning, sequencing, and functional expression of a cDNA encoding the human V1a vasopressin receptor, J. Biol. Chem. 269 (1994) 3304-3310. 
[47] M. Thibonnier, M.K. Graves, M.S. Wagner, C. Auzan, E. Clauser, H.F. Willard, Structure, sequence, expression, and chromosomal localization of the human V1a vasopressin receptor gene, Genomics 31 (1996) 327-334.

[48] J.D. Thompson, D.G. Higgins, T.J. Gibson, CLUSTAL W: improving the sensitivity of progressive multiple sequence alignment through sequence weighting, position-specific gap penalties and weight matrix choice, Nucleic Acids Res. 22 (1994) 4673-4680.

[49] R.R. Thompson, J.C. Walton, Peptide effects on social behavior: effects of vasotocin and isotocin on social approach behavior in male goldfish (Carassius auratus), Behav. Neurosci. 118 (2004) 620-626.

[50] M.A. Ventura, P. René, Y. de Keyzer, X. Bertagna, E. Clauser, Gene and cDNA cloning and characterization of the mouse V3/V1b pituitary vasopressin receptor, J. Mol. Endocrinol. 22 (1999) 251-260.

[51] H. Walum, L. Westberg, S. Henningsson, J.M. Neiderhiser, D. Reiss, W. Igl, J.M. Ganiban, E.L. Spotts, N.L. Pedersen, E. Eriksson, P. Lichtenstein, Genetic variation in the vasopressin receptor 1a gene (AVPR1A) associates with pair-bonding behavior in humans, Proc. Natl. Acad. Sci. USA 105 (2008) 14153-14156.

[52] A.M. Waterhouse, J.B. Procter, D.M.a. Martin, M. Clamp, G.J. Barton, Jalview Version 2--a multiple sequence alignment editor and analysis workbench, Bioinformatics 25 (2009) 1189-1191.

[53] P.J. Zak, A.A. Stanton, S. Ahmadi, Oxytocin increases generosity in humans, PLoS ONE 2 (2007) e1128. 


\section{FIGURE 1}

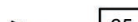

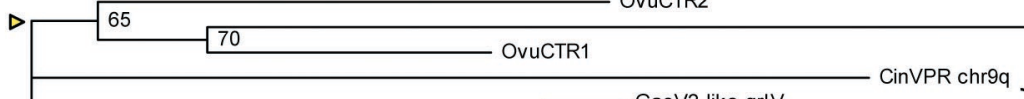
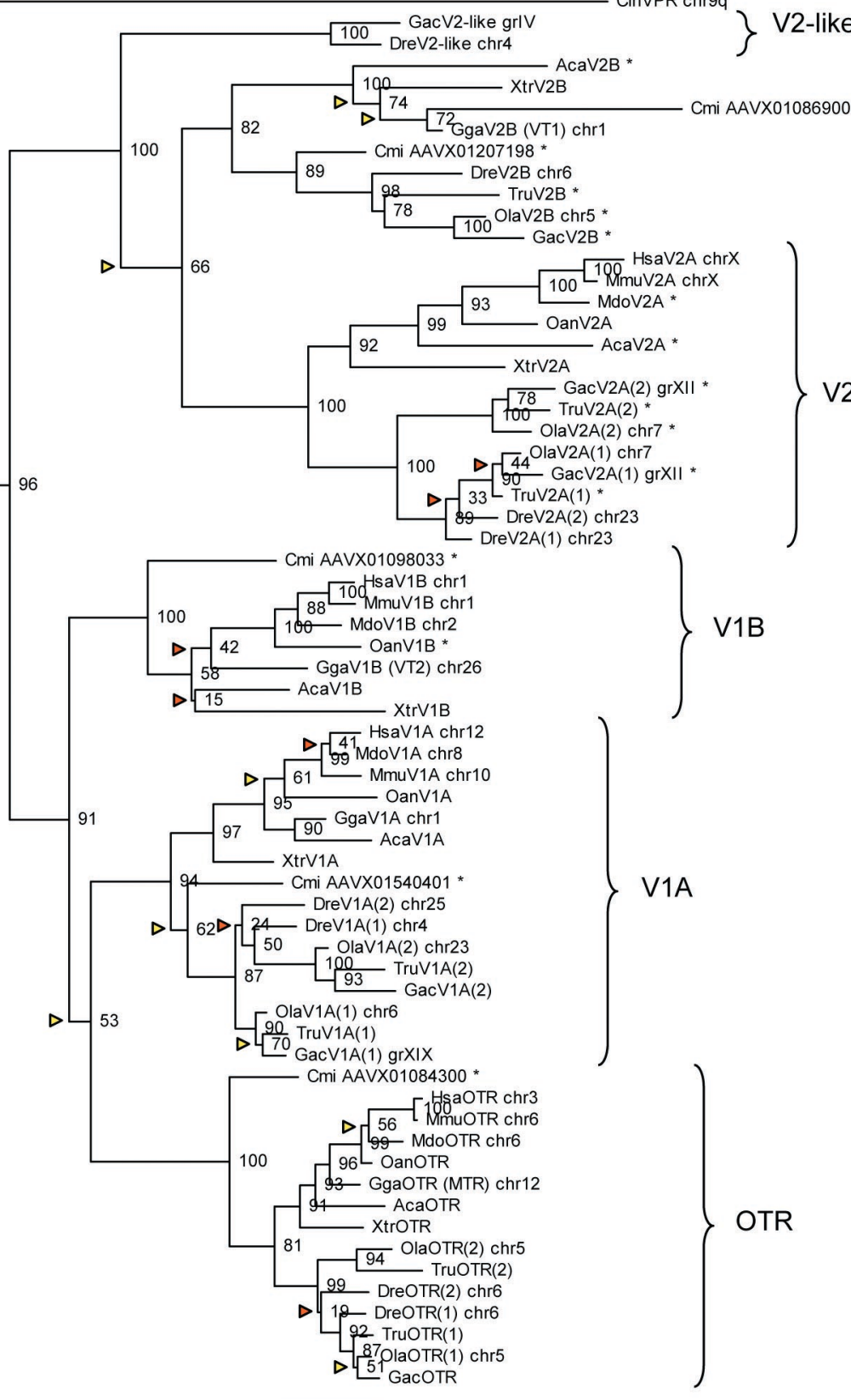
